\title{
Procesos de aprendizaje adulto en contextos de educación no formal ${ }^{*}$
}

\section{Adult Learning Processes in Non-Formal Education Contexts}

Recepción: 01 Febrero 2016 | Aprobación: 09 Septiembre 2017

\author{
Celia Acero Pereira ${ }^{a}$ \\ Universidad de Sevilla, España \\ ORCID: http://orcid.org/0000-0002-6451-5981 \\ M. Victoria Hidalgo \\ Universidad de Sevilla, España \\ LuCÍA JIMÉNEZ \\ Universidad de Sevilla, España
}

a Autora de correspondencia. Correo electrónico:
celaceper@gmail.com

Para citar este artículo: Acero Pereira, C., Hidalgo, M. V., \& Jiménez, L. (2018). Procesos de aprendizaje adulto en contextos de educación no formal. Universitas Psychologica, 17(2), 1-10. https://doi. org/10.11144/Javeriana.upsy.17-2.paac

\section{RESUMEN}

La compresión de los procesos de aprendizaje durante la adultez requiere considerar tanto el contexto de aprendizaje como las características personales de los aprendices. En este marco, el estudio que se describe en este artículo explora los procesos de aprendizaje adulto en contextos no formales siguiendo el modelo de Kolb (1984), a la vez que se analiza su variabilidad en función de diferentes características personales de los participantes. Los resultados obtenidos confirmaron la existencia de una importante variabilidad en las formas de aprender de los adultos participantes en la investigación, y mostraron que algunas características personales como las condiciones socio-laborales y la trayectoria personal de riesgo guardaban relación con las diferencias observadas. El papel de estas características personales, así como de otras variables relacionadas con los adultos como aprendices, es discutido, a la vez que se destacan importantes implicaciones metodológicas a tener en cuenta cuando se planifican experiencias educativas no formales con personas adultas.

Palabras clave

aprendizaje adulto; educación no formal; estilo de aprendizaje.

\begin{abstract}
It is necessary to evaluate the learning context and individual characteristics of learners to understand the adult learning process. In this study, adult learning processes at non-formal situations according to Kolb's model (1984) are described, considering its variability according to individual characteristics. Variability in adult learning processes was found, with socio-laboral conditions and risk trajectories playing a relevant role. These results are discussed, and methodological implications for planning adult learning experiences are highlighted.

Keywords

adult learning; non formal education; learning style.
\end{abstract}

Las personas aprenden continuamente nuevas habilidades, pues el aprendizaje es parte protagonista e inseparable de 
la existencia humana, y viceversa (Delors, 1996; Sarrate, 2009). Consecuentemente, las políticas educativas llevan años apoyando la educación permanente y sus beneficios (Lancho, 2009; Requejo, 2003). A pesar de su importancia, las evidencias empíricas sobre el aprendizaje permanente son escasas y centradas en contextos formales. Buscando aportar nuevos datos empíricos en este campo, esta investigación aborda el estudio del aprendizaje adulto en experiencias de educación no formal.

Como etapa evolutiva, la adultez se caracteriza por el aumento y variedad de experiencias vitales, en las que los roles que cada persona desempeña son esenciales en la configuración de su identidad (Papalia \& Feldman, 2012). Consecuentemente, los procesos de aprendizaje adulto presentan ciertas peculiaridades: las personas parten de su bagaje vital, ven el aprendizaje como una herramienta útil para resolver sus necesidades, y no un fin en sí mismo, en donde demandan la utilidad práctica de lo aprendido; están motivados intrínsecamente, relacionan sus aprendizajes con el resto de roles desempeñados, y necesitan sentirse protagonistas de su aprendizaje (Pizarro, 2012; Undurraga, 2004). Probablemente estas características generales interactúan con las singularidades de cada trayectoria para matizar los procesos de aprendizaje. En esta línea, la evidencia indica que las peculiaridades del aprendizaje adulto no vienen determinadas tanto por la edad, como por la trayectoria vital (Jinkens, 2009; Wu \& Wu, 2015). Así, existen estudios que muestran que no solo las circunstancias actuales afectan a los resultados de aprendizaje; por ejemplo, un adulto con una historia previa de fracaso escolar afrontará nuevas experiencias de aprendizaje desde una posición de rechazo e inseguridad, especialmente cuanto más similar sea la situación de aprendizaje a esas primeras experiencias (Belzer, 2004; Freire, 1970). En este caso, el proceso de aprendizaje solo será exitoso si la persona tiene un proyecto vital definido y ve el aprendizaje como un instrumento para lograr sus objetivos (Blanco \& Rodríguez, 2015).

Por tanto, la investigación apunta que es importante conocer la trayectoria vital de los adultos para construir situaciones de aprendizaje exitosas, al menos en contextos académicos. Es difícil extrapolar tal conclusión a situaciones no formales e informales, ya que han sido poco estudiadas, a pesar de que es donde las personas adquieren y transfieren aprendizajes de manera más frecuente y efectiva (Ellinger, 2005; Marsick, 2003). Como excepción, el estudio de Kim y McLean (2014) confirma que las situaciones de aprendizaje informal en el ámbito laboral están influidas tanto por variables individuales como por el contexto y la cultura.

Entre las características individuales que marcan diferencias en los procesos de aprendizaje están las estrategias de aprendizaje utilizado (Willingham, 2014). Así, los estudiantes de mayor rendimiento utilizan más estrategias relacionadas con la búsqueda y organización de información en comparación con los de bajo rendimiento (Kormos \& Csizér, 2014; Nandagopal \& Ericsson, 2012).

Igualmente, el estilo influye en el aprendizaje y el rendimiento. Muchas teorías han tratado de definir qué son los estilos de aprendizaje y su funcionamiento (Coffield, Moseley, Hall, \& Ecclestone, 2004). Entre las más destacadas se encuentra el modelo de aprendizaje experiencial (ELT) de Kolb (1984), enmarcado en las teorías que conciben el estilo de aprendizaje desde una perspectiva flexible. Kolb define el aprendizaje como proceso y no como producto, planteamiento que se adecúa a las situaciones de aprendizaje no formal con adultos, objeto de este trabajo (Rodrigo, Máiquez, \& Martín, 2010).

La teoría de Kolb describe el aprendizaje como un ciclo de cuatro fases: experiencia concreta (EC); conceptualización abstracta (CA); observación reflexiva (OR); y experimentación activa (EA); agrupables en dos etapas complementarias (adquisición y transformación), que dan lugar a cuatro estilos de aprendizaje. Según Kolb (1984) la etapa de adquisición del conocimiento, que permite crear una representación mental de la realidad, puede basarse en la EC (acercamiento intuitivo y artístico, que busca la implicación en situaciones de contacto humano) y/o en la CA (aproximación científica, utilizando la 
lógica, las ideas y los conceptos). La etapa de transformación, donde se integra la nueva información con las ideas previas, puede basarse en la OR (centrada en comprender y conocer la realidad más que en aplicar los nuevos conocimientos) y/o en la EA (trata de influir y cambiar las situaciones con una finalidad pragmática). El proceso de aprendizaje ideal integra las cuatro fases, que representan un ciclo equilibrado. Sin embargo, es posible adquirir y organizar las experiencias de aprendizaje centrándose en alguna de las fases.

Este modelo permite distinguir cuatro estilos de aprendizaje, según la combinación de las cuatro fases. Las personas con estilo divergente $(\mathrm{EC}+\mathrm{OR})$ analizan cada situación desde diferentes perspectivas, observan antes de actuar y están orientadas hacia el contacto social. Las personas con estilo asimilador (CA $+\mathrm{OR}$ ) ordenan la información lógicamente hasta crear un modelo teórico propio; razonan inductivamente y están centradas en los conceptos abstractos. Las personas con estilo convergente $(\mathrm{CA}+\mathrm{EA})$ tienen la habilidad de descubrir la aplicación práctica de las ideas, resolver problemas y tomar decisiones. Finalmente, las personas con estilo acomodador (EC+EA) se implican en nuevas experiencias que conlleven acción y cambio, y actúan en función de sus sentimientos.

Muchas investigaciones han estudiado situaciones de aprendizaje adulto siguiendo el modelo de Kolb. La mayoría han sido realizadas con estudiantes universitarios (véase una revisión en Kolb \& Kolb, 1999), en donde no hay consenso sobre cuál es el estilo más habitual. Esta variabilidad se explica porque no siempre hay un único estilo estable para cada persona; con frecuencia existe un segundo estilo potencial que según las actividades podrá manifestarse en mayor o menor medida e incluso convertirse en el estilo principal (Narli, Ozgen, \& Alkan, 2011).

Los datos disponibles sobre el proceso de aprendizaje adulto, siguiendo la teoría de Kolb, se centran en alumnado universitario y en las características del individuo como aprendiz. La evidencia muestra que el estilo de aprendizaje marca diferencias en el rendimiento, la satisfacción y la preferencia por el tipo de metodología y/o contenido (Bhatti \& Bart, 2013; Sahasrabudhe \& Kanungo, 2014). Pero no hay datos para saber si tal relación se mantiene en contextos no formales. Además, carecemos de estudios que relacionen los procesos de aprendizaje con características personales más allá del rol como aprendiz.

Los escasos estudios existentes muestran relación entre el estilo de aprendizaje y el nivel de estudios, el área de especialización y la actividad laboral, al menos en contextos formales (Can, 2011; Kolb, 1984), aunque no hay consenso sobre el papel del sexo. Mientras que Kandil (2015) y Cavanagh (1995) no encontraron diferencias en el estilo de aprendizaje según el sexo, Heffler (2001) y Cavas (2010) sí lo hicieron, lo que no permite obtener conclusiones claras. Tampoco hay datos acerca de la relación entre el estilo y las estrategias de aprendizaje, o la trayectoria vital, a pesar de que ambas dimensiones desempeñan un papel relevante en el proceso de aprendizaje (Freire, 1970; Rogers, 2004).

La revisión realizada pone de manifiesto que la compresión de los procesos de aprendizaje durante la adultez requiere considerar tanto el contexto de aprendizaje como las características personales de los aprendices. Teniendo en cuenta que la mayor parte de la investigación sobre aprendizaje adulto se ha realizado en contextos académicos, este estudio se propone explorar los procesos de aprendizaje adulto en contextos no formales, examinando su variabilidad en función de diferentes características personales. En concreto, se pretende: (1) describir el proceso de aprendizaje adulto de personas que participan en una experiencia de aprendizaje no formal según el modelo de Kolb (1984); y (2) estudiar las diferencias en el proceso de aprendizaje en función del perfil sociodemográfico, de riesgo y las estrategias de aprendizaje de los participantes. 


\section{Método}

\section{Participantes}

Setenta y cinco adultos residentes en Perú, 44 de Leymebamba, y 31 de Cacatachi. Concretamente 63 mujeres (84\%) y 12 hombres (16\%); con una media de 36.89 años $(D T=8.47)$. Se distribuyeron entre estudios básicos (37.1\%), secundarios $(28.6 \%)$ y superiores $(34.3 \%)$. La mayoría trabajaba $(62.9 \%)$, de manera estable $(72.1 \%)$ y con baja o media cualificación (52.5\%). La mayoría pertenecían a una familia biparental (77.3\%). El nivel de riesgo psicosocial vivido indicó que la media de eventos estresantes fue de $2.19(D T=2,24)$

Se comprobó la comparabilidad del perfil sociodemográfico entre las dos zonas y ambos grupos resultaron comparables en sexo, perfil laboral y riesgo psicosocial. Solo hubo diferencias significativas en la edad y el nivel de estudios, donde los participantes de Leymebamba $(M=$ 40.35, DT $=8.9$ ) fueron de mayor edad que los de Cacatachi $(M=33.09, D T=6.43)$, y en mayor proporción con estudios básicos (46.2\%) frente a secundarios $(48.4 \%)$.

\section{Instrumentos}

Inventario de Situaciones Estresantes y de Riesgo, ISER (Hidalgo et al., 2005): Define el perfil de riesgo psicosocial de acuerdo a 16 situaciones (e.g., "ser víctima de maltrato"), evaluando si una persona o alguien cercano las ha vivido en los últimos tres años; así como el nivel de afectación en cada caso (de $1=$ poco a 3 = mucho). Se obtienen tres medidas para la acumulación de factores de riesgo a nivel individual, familiar y global (individual + familiar); y tres para la afectación correspondiente.

Kolb, Learning Style Inventory LSI 3.1 (Kolb, 2007): Define el estilo de aprendizaje mediante 12 ítems con cuatro opciones de respuesta que se deben ordenar desde la que más describe el propio proceso de aprendizaje hasta la que menos. Cada opción se corresponde con una de las cuatro fases del proceso de aprendizaje: "Cuando estoy aprendiendo: soy una persona observadora (OR), soy una persona activa (EA), soy una persona intuitiva (EC), soy una persona lógica (CA)". Aunque permite recoger tres medidas, para este estudio solo se utilizaron dos. Por un lado, una valoración cuantitativa (en una escala de 1 a 4) del proceso de aprendizaje, que informa sobre el énfasis relativo que una persona da a cada una de las cuatro fases (OR frente a EA, que componen la etapa de adquisición y EC frente a CA, que componen la etapa de transformación). Por otro lado, una valoración cualitativa del estilo de aprendizaje (divergente, asimilador, convergente o acomodador), fruto de combinar las puntuaciones anteriores.

Estrategias de aprendizaje (ad hoc): Entrevista en torno a dos situaciones cotidianas de crianza de los hijos para las que se realizaban tres preguntas ("Cómo podría resolver la situación tu amiga/o? Y si lo intenta y no le sale, ¿cómo puede aprender? ¿Qué has hecho tú para aprender a actuar en este tipo de situaciones?"), cuyas respuestas se clasificaron en estrategias académicas (e.g., "He aprendido leyendo algunos libros") y cotidianas (e.g., "Aprendí por medio de mi tía, porque yo lo veía cómo ella lo trataba a su niño").

\section{Procedimiento}

En el marco de un proyecto de cooperación al desarrollo entre España y Perú, se desarrolló una intervención de aprendizaje no formal para adultos, en concreto, un programa de educación parental. El 90 \% de los participantes colaboraron voluntariamente en la investigación. Se consideraron las recomendaciones de la APA (2011) sobre los aspectos éticos. La evaluación se realizó de manera individual y en formato entrevista.

\section{Análisis de datos}

Para el estudio de los datos cualitativos se aplicó un análisis de contenido. El universo objeto de estudio lo conformaron las respuestas de la 
entrevista (véase el apartado de instrumentos). En la codificación de respuestas se siguieron los principios de exhaustividad y exclusividad y se realizaron comprobaciones de coherencia, donde se establecieron dos categorías: estrategias de aprendizaje académicas y cotidianas.

Para el análisis descriptivo de las variables cuantitativas se presentaron la media, la desviación típica y los valores máximos y mínimos; para las variables cualitativas, las frecuencias y los porcentajes. Para los contrastes se realizaron análisis de medidas repetidas, contingencia y varianza, utilizando Chi cuadrado y F de Sneidecor, respectivamente. Para los análisis de contingencia se utilizó la $\mathrm{V}$ de Cramner como estadístico del tamaño del efecto; en los análisis de varianza y de medidas repetidas, $\eta^{2}$ parcial. Se comprobaron los supuestos pertinentes y la presencia de casos extremos siguiendo las recomendaciones de Hair, Anderson, Tatham, y Black (2008), con resultados satisfactorios. Se empleó SPSS versión 18.

\section{Resultados}

Proceso de aprendizaje adulto: caracterización según las fases y los estilos de aprendizaje

El examen del proceso de aprendizaje adulto mostró que la fase más característica en este grupo fue EA, con una media de $2.63(\mathrm{DT}=0.4)$. La puntuación media para CA fue $2.58(\mathrm{DT}=$ 0.39), para $E C$ fue $2.35(D T=0.39)$ y para $O R$ fue $2.44(D T=0.56)$. El estilo de aprendizaje más frecuente fue el divergente, característico para 27 personas (36\%), 18 adultos presentaron estilo asimilador (24\%), 15 estilo convergente (20\%) y 15 acomodador (20\%).
Proceso de aprendizaje en función del perfil sociodemográfico de los participantes

\section{Tabla 1}

Descriptivos de las fases de aprendizaje en función del perfil sociodemográfico

\begin{tabular}{|c|c|c|c|c|c|c|c|c|c|}
\hline & & \multicolumn{2}{|c|}{$\begin{array}{l}\text { Experiencia } \\
\text { concreta }\end{array}$} & \multicolumn{2}{|c|}{$\begin{array}{c}\text { Conceptualización } \\
\text { abstracta }\end{array}$} & \multicolumn{2}{|c|}{$\begin{array}{l}\text { Observación } \\
\text { reflexiva }\end{array}$} & \multicolumn{2}{|c|}{$\begin{array}{c}\text { Experimentación } \\
\text { activa }\end{array}$} \\
\hline & & & & & & & $D T$ & & \\
\hline \multirow[t]{2}{*}{ Sexo } & Hombre & 2.4 & 0.32 & 2.66 & 0.43 & 2.31 & 0.57 & 2.63 & 0.47 \\
\hline & Mujer & 2.33 & 0.41 & 2.56 & 0.39 & 2.47 & 0.56 & 2.66 & 0.39 \\
\hline \multirow[t]{3}{*}{ Edad } & $20-35$ años & 2.31 & 0.46 & 2.56 & 0.38 & 2.39 & 0.58 & 2.74 & 0.42 \\
\hline & $36-50$ aid & 2.3 & 0.32 & 2.5 & 0.4 & 2.56 & 0.58 & 2.55 & 0.4 \\
\hline & $51-65$ & 2.53 & 0.1 & 2.7 & 0.39 & 2.08 & 0.43 & 2.64 & 0.13 \\
\hline \multirow{3}{*}{$\begin{array}{l}\text { Nivel de } \\
\text { estudios }\end{array}$} & Básicos & 2.36 & 0.35 & 2.49 & 0.36 & 2.58 & 0.62 & 2.58 & 0.46 \\
\hline & 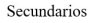 & 2.25 & 0.5 & 54 & 0.44 & 2.63 & 0.51 & 2.58 & 0.37 \\
\hline & Super & 2.4 & 0.33 & 2.72 & 0.33 & 2.16 & 0.47 & 2.72 & 0.37 \\
\hline Situación & Inacti & 2.2 & 0.43 & 63 & 37 & 61 & 0.55 & .57 & 0.38 \\
\hline laboral & & 2.44 & 0.3 & & & & 0.58 & & 0.42 \\
\hline alificas & Baja o & 2.41 & 0.3 & 2.3 & 37 & 58 & 0.6 & 2.65 & .51 \\
\hline laboral & Alta & 2.45 & 0.3 & 2.79 & 32 & 06 & 0.39 & 2.7 & 0.33 \\
\hline Estabilid & 10 & 2.42 & 0. & & 0.35 & 31 & 0.59 & 86 & 0.43 \\
\hline laboral & Si & 2.37 & 0.3 & 2. & 0.4 & 2.4 & 0.6 & 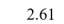 & .41 \\
\hline & $\mathrm{N}$ & 2.37 & 0. & & & & & 2.53 & 37 \\
\hline & $\mathrm{Si}$ & 2.3 & 0.4 & & 0.35 & & 0.5 & 2.7 & 0.43 \\
\hline & par & 2.3 & 0.4 & 2.62 & 0.4 & 2.41 & 0.57 & 2.67 & 0.41 \\
\hline familiar & Monoparental & 2.48 & 0.37 & 2.35 & 0.24 & 2.77 & 0.05 & 2.41 & 0.39 \\
\hline
\end{tabular}

El análisis de las cuatro fases del aprendizaje en función del perfil sociodemográfico (véase Tabla 1) muestra en relación con EC, diferencias en función de la situación laboral a favor de las personas que trabajaban $(F(1,68)=5.72, p=$ $0.02, \eta^{2}$ parcial $\left.=0.08\right)$. En cuanto a la fase de CA, las personas que trabajaban en puestos de alta cualificación presentaron mayor CA que las de baja o media cualificación, $(F(1,48)=15.03$, $p=0, \eta^{2}$ parcial $\left.=0.28\right)$. Lo mismo ocurre para personas con ingresos estables frente a ingresos no estables $\left(F(1,62)=4.57, p=0.037, \eta^{2}\right.$ parcial $=0.07)$, y para familias biparentales frente a monoparentales $\left(F(1,48)=4.44, p=0.039, \eta^{2}\right.$ parcial $=0.06)$. Atendiendo a la $\mathrm{OR}$, se hallaron diferencias en relación al nivel de estudios $(F(2$, 67) $=5.35, p=0.007, \eta^{2}$ parcial $\left.=0.14\right)$; quienes tenían estudios secundarios o estudios básicos mostraron una mayor $\mathrm{OR}$ que quienes tenían estudios superiores. Lo mismo ocurrió con las personas con un trabajo de baja o media cualificación frente a alta cualificación $(F(1,38)$ $=9.55, p=0.004, \eta^{2}$ parcial $\left.=0.2\right) ; y$ con quienes tenían ingresos inestables en comparación con quienes tenían ingresos estables $(F(1,62)=4.52$, $p=0.037, \eta_{\text {parcial }}^{2}=0.07$ )

Atendiendo a los cuatro estilos de aprendizaje, se hallaron diferencias en función de la situación laboral, la estabilidad de ingresos y la estructura 
familiar (véase Tabla 2). Entre las personas que no trabajan destacaba una mayor frecuencia de estilo de aprendizaje asimilador $\left(X^{2}(3, N=70)=\right.$ $\left.11.7, p=0.008 ; V_{\text {cramner }}=0.41\right)$. En cuanto a la estructura familiar, un gran porcentaje de adultos de familias monoparentales presentaban un estilo divergente, mientras que ninguno de ellos mostró un estilo asimilador $\left(\mathrm{X}^{2}(3, \mathrm{~N}=68)=10.73, p\right.$ $\left.=0.013 ; V_{\text {cramner }}=0.4\right)$. Entre las personas con inestabilidad de ingresos, en escasas ocasiones se empleaba un estilo convergente $\left(\mathrm{X}^{2}(3, \mathrm{~N}=64)\right.$ $\left.=9.92, p=0.019 ; V_{\text {cramner }}=0.39\right)$.

\section{Tabla 2}

Descriptivos del estilo de aprendizaje en función del perfil (porcentajes)

\begin{tabular}{llrrrr}
\hline & & Divergente & Asimilador & Convergente & Acomodador \\
\hline Sexo & Hombre & 41.7 & 8.3 & 33.3 & 16.7 \\
& Mujer & 34.9 & 27 & 17.5 & 20.6 \\
\hline Edad & 20-35 años & 26.7 & 26.7 & 23.3 & 23.3 \\
& 36-50 años & 40.6 & 25 & 18.8 & 15.6 \\
& 51-65 años & 33.3 & 33.3 & 33.3 & 0 \\
\hline Nivel de & Básico & 34.6 & 26.9 & 19.2 & 19.2 \\
estudios & Secundario & 40 & 35 & 5 & 20 \\
& Superior & 33.3 & 12.5 & 37.5 & 16.7 \\
\hline Situación & Inactivo & 30.8 & $\mathbf{4 6 . 2}$ & 15.4 & 7.7 \\
laboral & Activo & 38.6 & 11.4 & 25 & 25 \\
\hline Cualificación & Baja o media & 47.6 & 9.5 & 9.5 & 33.3 \\
laboral & Alta & 26.3 & 15.8 & 42.1 & 15.8 \\
\hline Estabilidad & No & 25 & 8.3 & 25 & 41.7 \\
laboral & Si & 41.9 & 16.1 & 25.8 & 16.1 \\
\hline Estabilidad & No & 46.4 & 28.6 & $\mathbf{3 . 6}$ & 21.4 \\
ingresos & Si & 27.8 & 22.2 & 36.1 & 13.9 \\
\hline Tipo de & Biparental & 27.6 & 29.3 & 24.1 & 19 \\
familia & Monoparental & $\mathbf{8 0}$ & $\mathbf{0}$ & 10 & 10 \\
\hline & & & & & \\
\hline
\end{tabular}

El proceso de aprendizaje en función del perfil de riesgo de los participantes

En la Tabla 3 se ponen en relación las cuatro fases del ciclo de aprendizaje con el perfil de riesgo: la acumulación de factores de riesgo y la afectación asociada al nivel individual, familiar y global. Se encontraron diferencias significativas en función de la afectación asociada al nivel de riesgo vivido por la propia persona entrevistada. Las personas con un nivel medio de afectación presentaron mayor $\mathrm{EC}$ que las personas con una afectación elevada $\left(F(2,35)=3.71, p=0.035, \eta^{2}\right.$ parcial $=0.18)$; y las personas con un nivel alto de afectación mayor $\mathrm{CA}$ que las personas con un nivel medio $(F(2,35)=3.76, p=0.034$, $\eta^{2}$ parcial $\left.=0.18\right)$. En relación con la EA, se observaron diferencias para el nivel de riesgo en el entorno cercano y de acuerdo al riesgo global. Estas diferencias fueron a favor de quienes tuvieron un nivel alto de riesgo en su entorno frente a quienes tuvieron un nivel medio ( $F(1$, $35)=3.58, p=0.039, \eta^{2}$ parcial $\left.=0.17\right)$; $\mathrm{y}$ a favor de las personas con un nivel de riesgo global alto frente a las personas con un nivel de riesgo global medio $\left(F(1,36)=2.93, p=0.067, \eta^{2}\right.$ parcial $=$ $0.15)$.

\section{Tabla 3}

Estadísticos descriptivos de las fases de aprendizaje en función del perfil de riesgo

\begin{tabular}{|c|c|c|c|c|c|c|c|c|c|}
\hline & & \multicolumn{2}{|c|}{$\begin{array}{l}\text { Experiencia } \\
\text { concreta }\end{array}$} & \multicolumn{2}{|c|}{$\begin{array}{c}\text { Conceptualización } \\
\text { abstracta }\end{array}$} & \multicolumn{2}{|c|}{$\begin{array}{l}\text { Observación } \\
\text { reflexiva }\end{array}$} & \multicolumn{2}{|c|}{$\begin{array}{l}\text { Experimentación } \\
\text { activa }\end{array}$} \\
\hline & & $M$ & $D T$ & $M$ & $D T$ & $M$ & $D T$ & $M$ & $D T$ \\
\hline \multirow{3}{*}{$\begin{array}{l}\text { Riesgo } \\
\text { individual }\end{array}$} & Bajo & 2.34 & 0.37 & 2.6 & 0.35 & 2.38 & 0.55 & 2.69 & 0.38 \\
\hline & Medio & 2.42 & 0.42 & 2.4 & 0.35 & 2.7 & 0.58 & 2.48 & 0.47 \\
\hline & Alto & 2.33 & 0.6 & 2.36 & 0.56 & 2.69 & 0.77 & 2.62 & 0.42 \\
\hline \multirow{3}{*}{$\begin{array}{l}\text { Riesgo } \\
\text { entorno }\end{array}$} & Bajo & 2.36 & 0.36 & 2.59 & 0.36 & 2.31 & 0.59 & 2.73 & 0.39 \\
\hline & & 42 & 0.39 & 2.57 & 0.41 & 2.49 & 0.44 & 2.52 & 0.25 \\
\hline & Alto & 45 & 0.49 & 2.63 & & 1.92 & 0.54 & 3 & 0.26 \\
\hline \multirow{3}{*}{$\begin{array}{l}\text { Riesgo } \\
\text { global }\end{array}$} & Bajo & 8 & 0.33 & 2.6 & 5 & 2.29 & 0.49 & 2.72 & 0.35 \\
\hline & M & 36 & 0.46 & 2.64 & 0.41 & 2.51 & 0. & 2.5 & 0.31 \\
\hline & Alto & 2.48 & 0.52 & 2.42 & 0.55 & 2.17 & 0.54 & 2.93 & 0.34 \\
\hline \multirow{3}{*}{$\begin{array}{l}\text { Afectación } \\
\text { individual }\end{array}$} & Baj & 2.36 & & & & 2.29 & & 2.7 & 0.37 \\
\hline & Medi & 2.73 & 0.19 & & & 2.2 & & 2.88 & 0.27 \\
\hline & Alto & 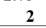 & & & & 2.92 & & 2.42 & 0.35 \\
\hline \multirow{3}{*}{$\begin{array}{l}\text { Afectación } \\
\text { entorno }\end{array}$} & Bajo & 2.38 & & & & 2.38 & & 2.67 & 0.37 \\
\hline & Med & 2.4 & 0. & 2.3 & & 2.25 & 0.3 & 2.78 & 0.35 \\
\hline & Alto & 2.5 & 0.29 & 2.83 & 0.2 & 1.75 & 0.4 & 2.92 & 0.3 \\
\hline \multirow{3}{*}{$\begin{array}{l}\text { Afectación } \\
\text { total }\end{array}$} & Bajo & 2.3 & & & & 2.34 & 0.5 & 2.66 & 0.37 \\
\hline & Medi & 2.43 & 0.46 & 2.48 & 0.41 & 2.26 & 0.6 & 2.83 & 0.34 \\
\hline & Alto & 2.55 & 0.35 & 2.47 & 0.51 & 2.17 & 0.63 & 2.81 & J.3 \\
\hline
\end{tabular}

Al estudiar la relación entre el perfil de riesgo y los estilos de aprendizaje, se agrupó la variable riesgo en dos valores (bajo y medio-alto), cumpliendo así los requerimientos de la prueba Chi cuadrado (Hair et al., 2008). No se hallaron diferencias significativas.

El proceso de aprendizaje en función del tipo de estrategias de aprendizaje

En cuanto a las fases del proceso de aprendizaje en función de las estrategias de aprendizaje empleadas, las personas que recurrían en su mayoría a estrategias de aprendizaje cotidianas mostraron mayor EC $(M=2.49=2.22 ; F(1,33)$ $=4.54, p=0.041, \eta^{2}$ parcial $\left.=0.12\right)$; mientras que quienes utilizaron mayoritariamente estrategias académicas tuvieron mayor CA $(\mathrm{M}=2.76, \mathrm{DT}$ $=0.37)$, aunque con significación marginal $(F(1$, $33)=3.28, p=0.079, \eta^{2}$ parcial $\left.=0.09\right)$. No se hallaron relaciones significativas entre el estilo y las estrategias de aprendizaje. 


\section{Discusión}

Esta investigación tenía un doble objetivo, describir el proceso de aprendizaje adulto de personas que participan en una experiencia de aprendizaje no formal según el modelo de Kolb (1984), y estudiar posibles diferencias en función de algunas características personales.

Respecto al primer objetivo, los adultos del estudio presentaron mayoritariamente un estilo divergente. Aunque la mayor prevalencia de este estilo, caracterizado por la presencia de respuestas creativas y que suponen el contacto entre personas, coincide con lo encontrado en otros estudios con población universitaria (Cavas, 2010), en conjunto los resultados destacan fundamentalmente la existencia de una importante variabilidad en las formas de aprender de los adultos participantes. En concreto, los resultados muestran una distribución bastante homogénea tanto en los cuatro estilos como en las cuatro fases del modelo de Kolb (1984). Probablemente esta variabilidad se relacione con el hecho de que las personas muestran diferencias en cómo aprenden en función de la tarea de aprendizaje, donde manifiestan \#de acuerdo a la situación\# un estilo u otro (Narli et al., 2011). Además, las características de la etapa adulta, con mayores diferencias individuales que en otros períodos, pueden contribuir a explicar la variabilidad observada (Papalia \& Feldman, 2012).

La necesidad de explorar y explicar la variabilidad observada en los procesos de aprendizaje adulto constituye el segundo objetivo de este trabajo. Los resultados muestran que algunas de las características personales de los adultos participantes se relacionan con sus procesos de aprendizaje; en concreto, fueron las condiciones sociolaborales (especialmente el nivel de cualificación y la estabilidad laboral y económica), junto a la trayectoria vital de riesgo las dimensiones que establecieron más diferencias. Así, las personas con mejores condiciones sociolaborales se caracterizaron en mayor medida por un estilo convergente y una mayor CA en la etapa de adquisición de nuevos conocimientos, lo que supone una aproximación científica y lógica al contenido a aprender. Como está bien documentado, las personas con unas condiciones laborales más positivas suelen caracterizarse por una trayectoria de éxito académico que ha podido influir en su proceso de aprendizaje (Freire, 1970; Rogers, 2004), lo que les proporciona más oportunidades para el desarrollo de un pensamiento lógico, característico de la fase denominada por Kolb (1984) como CA. En cambio, se halló que las personas con una trayectoria de mayor riesgo psicosocial se caracterizaron por una mayor presencia de EA en la etapa de adquisición de nuevos conocimientos. Este tipo de aproximación al aprendizaje pone el acento en la utilidad práctica de los conocimientos, en consonancia con las características generales del adulto como aprendiz, descritas en la introducción (Pizarro, 2012). Se trata de una forma de aprender que puede resultar particularmente adaptativa en situaciones de vulnerabilidad y riesgo, si tenemos en cuenta las complejas circunstancias que afrontan estas personas (Rodrigo, Máiquez, Martín, \& Byrne, 2008). Existen otros estudios que han puesto de manifiesto cómo estas trayectorias vitales de riesgo impregnan los procesos de aprendizaje. Así, Wu y Wu (2015) comprobaron que las mujeres que sufrían opresión en sus vidas, asumían este mismo rol también en el ámbito académico. Menos esperables son los resultados encontrados en relación a las características de las personas que en mayor medida se definen por la utilización de la OR en la etapa de transformación. En concreto, se trataba de adultos que desempeñaban trabajos de menor cualificación y tenían mayor inestabilidad económica. Este resultado puede ser contradictorio con lo discutido en relación a la etapa de adquisición y pone el acento en la necesidad examinar de forma diferencial las distintas fases propias del proceso de aprendizaje (Kolb, 1984). Finalmente, los resultados del estudio ponen de relieve la variabilidad de los procesos de aprendizaje en función de otras características de los adultos como aprendices. Concretamente, los adultos que empleaban más estrategias cotidianas 
adquirían el conocimiento fundamentalmente mediante experimentación concreta, mientras que quienes utilizaban estrategias académicas se caracterizaban por un mayor uso de la conceptualización abstracta, confirmando el papel que desempeñan estas estrategias en el proceso de aprendizaje (Willingham, 2014). Es decir, las personas que para adquirir nuevos conocimientos emplean un acercamiento intuitivo e interactivo se caracterizan, a su vez, por estrategias relacionadas con el contacto social y la creatividad. Igualmente, quienes adquieren nuevos conocimientos mediante una aproximación lógica y conceptual emplean, a su vez, estrategias que implican la búsqueda de un conocimiento experto y veraz.

En definitiva, en esta investigación se presenta información novedosa acerca de los procesos de aprendizaje de adultos en contextos no formales, un ámbito de intervención que va ganando reconocimiento, del que, sin embargo, no contamos con suficiente evidencia empírica. Además, frente a una aproximación más habitual, centrada en las características del proceso de enseñanza (e.g., Van Doorn, McManus, \& Yiend, 2012), este estudio pone el acento en el papel que desempeñan las características personales, tanto aquellas más individuales como otras propias del proceso de aprendizaje. No obstante, este trabajo no está exento de limitaciones, como haber contado con una muestra reducida que ha impedido realizar análisis multivariantes con suficientes garantías. Así mismo, podría haber resultado interesante incorporar algunas características del proceso de enseñanza para estudiar su papel interactivo con las características de aprendizaje de forma conjunta.

A pesar de las limitaciones señaladas, las conclusiones extraídas en esta investigación conllevan implicaciones prácticas relevantes. Por un lado, la variabilidad hallada en la forma de aprender de los adultos se traduce en la necesidad de que las propuestas educativas que se pongan en marcha incluyan suficiente diversidad de técnicas de enseñanza, apertura y flexibilidad para garantizar una óptima adquisición, y transformación de los contenidos por parte de todos los aprendices (Cubero, 2005). Por otro lado, los resultados obtenidos por los aprendices de condiciones sociolaborales desfavorables, y con una trayectoria vital de riesgo psicosocial, nos permiten concluir que estas personas se benefician de un aprendizaje dirigido a la utilidad de los nuevos conocimientos, y centrado tanto en la experiencia concreta como en la interacción con otros. Se trata de resultados que, a nivel empírico, confirman los principios metodológicos experienciales que desde presupuestos teóricos se defienden en la actualidad para la intervención psicoeducativa con adultos en general, y en situaciones de riesgo psicosocial, en particular (Rodríguez, Rodrigo, Martín, \& Máiquez, 2005). Este tipo de metodología se caracteriza por la reflexión cooperativa, el intercambio y el contraste de perspectivas como elementos conductores del aprendizaje, que promueven el entrenamiento reflexivo con el objeto de desarrollar conocimientos prácticos compartidos (Jiménez \& Hidalgo, 2012).

\section{Referencias}

American Psychological Association. (2011). Manual de publicaciones de la APA: guía de entrenamiento para el estudiante. México D. F.: American Psychological Association.

Belzer, A. (2004). "It's not like normal school": The role of prior learning contexts in adult learning. Adult Education Quarterly, 55(1), 41-59.

Bhatti, R., \& Bart, W. (2013). On the effect of learning styles on the scholastic achievement. Current Issues in Education, 16(2), 1-6.

Blanco, N., y Rodríguez, C. (2015) Actitud y compromiso hacia la escuela en estudiantes de secundaria considerados de éxito escolar. Infancia y Aprendizaje, 38(3), 542-568.

Can, S. (2011). Investigation of the relationships between the learning styles of preservice elementary teachers and some variables. Journal of Education, 41, 70-82.

Cavas, B. (2010). A study on pre-service science, class and mathematics teachers' 
learning styles in Turkey. Science Education International, 21(1), 47-61.

Cavanagh, S. (1995). The assessment of student nurse learning styles using the kolb learning styles inventory. Nurse Education Today, 15(3), 177-183.

Coffield, F., Moseley, D., Hall, E., \& Ecclestone, K. (2004). Learning styles and pedagogy in post-16 learning. A systematic and critical review. Recuperado de http://sxills.nl.

Cubero, R. (2005). Perspectivas constructivistas. La intersección entre el significado, la interacción y el discurso. Barcelona: Graó.

Delors, J. (1996). La educación encierra un tesoro. Informe de la Comisión Internacional sobre educación para el siglo XXI. Madrid: Santillana.

Ellinger, A. (2005). Contextual factors influencing informal learning in a workplace setting: the case of reinventing itself company. Human Resource Development, 16(3), 389-415.

Freire, P. (1970). Pedagogía del oprimido (19a ed.). Madrid: Siglo XXI.

Hair, J., Anderson, R., Tatham, R., \& Black, W. (2008). Análisis multivariante. Madrid: Prentice Hall.

Heffler, B. (2001). Individual learning style and the learning style inventory. Educational Studies, 27(3), 307-316.

Hidalgo, M. V., Menéndez, S., Sánchez, J., López, I., Jiménez, L., Lorence, B. (2005). 'Inventario de Situaciones Estresantes y de Riesgo (ISER)'. Documento sin publicar. Sevilla: Universidad de Sevilla.

Jiménez, L., \& Hidalgo, M. V. (2012). ¿Cómo se explica el cambio en las familias que participan en programas de formación y apoyo familiar? Sistemas Familiares, 28(1), $7-24$.

Jinkens, R. (2009). Nontraditional students: who are they? College Student, 43(4), 979-987.

Kandil, S. (2015). Turkish pre-service physics teachers' preferred learning styles. Educational Research and Reviews, 10(4), 403-415.

Kim, S., \& McLean, G. (2014). The impact of national culture on informal learning in the workplace. Adult Education Quarterly: A Journal of Research and Theory, 64 (1), 39-59.

Kolb, A., \& Kolb, D. (1999). Bibliography of research on experiential learning theory and the Learning Style Inventory. Cleveland: Case Western Reserve University.

Kolb, D. (1984). Experiential learning: Experience as a source of learning and development. Upper Saddle River: Prentice Hall.

Kolb, D. (2007). The Kolb Learning Style Inventory 3.1. Boston: Hay Learning.

Kormos, J., \& Csizér, K. (2014). The interaction of motivation, self-regulatory strategies, and autonomous learning behavior in different learner groups. TESOL, 48(2), 275-299.

Lancho, J. (2009). La educación de adultos, problemas y retos de futuro. En Educación de personas adultas en las fuerzas armadas (pp. 27-40). Madrid: Ministerio de Defensa.

Marsick, V. (2003). Invited reaction: Informal learning and the transfer of learning: How managers develop proficiency. Human Resource Development Quarterly, 14(4), 389-395.

Nandagopal, K., \& Ericsson, A. (2012). An expert performance approach to the study of individual differences in selfregulated learning activities in upper-level college students. Learning and Individual Differences, 22 (5), 597-609.

Narli, S., Ozgen, K., \& Alkan, H. (2011). In the context of multiple intelligences theory, intelligent data analysis of learning styles was based on rough set theory. Learning and Individual Differences, 21(5), 613-618.

Papalia, D., \& Feldman, R. (2012). Desarrollo Humano. México, D.F.: McGraw-Hill.

Pizarro, T. (2012). De la pedagogía a la andragogía en la educación superior. Revista Caribeña de Ciencias Sociales, 3, 1-31.

Requejo, A. (2003).Educación permanente y educación de adultos. Barcelona: Ariel.

Rodrigo, M. J., Máiquez, M. L., Martín, J. C., \& Byrne, S. (2008). Preservación familiar: un enfoque positivo para la intervención con familias. Madrid: Pirámide. 
Rodrigo, M., Máiquez, M., \& Martín, J. (2010). La educación parental como recurso psicoeducativo para promover la parentalidad positiva. Madrid: FEM.

Rodríguez, G., Rodrigo, M. J., Martín, J. C., \& Máiquez, M. L. (2005). El asesoramiento a familias con riesgo psicosocial. En J. I. Pozo, La práctica del asesoramiento educativo a examen (pp. 139-152). Barcelona: Graó.

Rogers, R. (2004). Storied selves: A critical discourse analysis of adult learners' iterate lives. Reading Research Quarterly, 39(3), 272-305.

Sarrate, M. (2009). Rasgos singulares del aprendizaje adulto. En Educación de personas adultas en las fuerzas armadas (pp. 27-40). Madrid: Gráficas.

Sahasrabudhe, V., \& Kanungo, S. (2014). Appropriate media choice for e-learning effectiveness: Role of learning domain and learning style. Computers $\mathcal{E}$ Education, 76, 237-249.

Undurraga, C. (2004). ¿Cómo aprenden los adultos? Santiago de Chile: Universidad Católica.

Van Doorn, K., McManus, F., \& Yiend, J. (2012). An analysis of matching cognitivebehavior therapy techniques to learning styles. Journal of Behavior Therapy and Experimental Psychiatry, 43(4), 1039-1044.

Willingham, D. (2014). Strategies make learning last. Educational Leadership, 72(2), 10-15.

Wu, Y., \& Wu, H. (2015). Higher education learning experiences among Vietnamese immigrant women in Taiwan. Adult Education Quarterly: A Journal of Research and Theory, 65(2), 133-151.

\section{Notas}

* Artículo de investigación. 